\title{
The effectiveness of microencapsulated sodium butyrate at reducing symptoms in patients with irritable bowel syndrome
}

\author{
Konrad Lewandowski ${ }^{1}$, Magdalena Kaniewska ${ }^{1}$, Katarzyna Karłowicz ${ }^{1}$, Mariusz Rosołowski ${ }^{2,3}$, \\ Grażyna Rydzewska ${ }^{1,4}$
}

\author{
${ }^{1}$ Clinical Department of Internal Medicine and Gastroenterology with Inflammatory Bowel Disease Unit, Central Clinical \\ Hospital of the Ministry of the Inferior and Administration, Warsaw, Poland \\ 2Department of Internal Medicine and Hypertension, Medical University of Bialystok, Bialystok, Poland \\ ${ }^{3}$ Department of Hypertension, Gastroenterology and Internal Medicine, Medical University of Bialystok Clinical Hospital, \\ Bialystok, Poland \\ ${ }^{4}$ Collegium Medicum, Jan Kochanowski University, Kielce, Poland
}

Key words: irritable bowel syndrome, symptoms, overweight, sodium butyrate.

\begin{abstract}
Address for correspondence: Magdalena Kaniewska MD, PhD, Clinical Department of Internal Medicine and Gastroenterology with Inflammatory Bowel Disease Unit, Central Clinical Hospital of the Ministry of the Inferior and Administration, 137 Wołoska St, 02-507 Warsaw, Poland, phone: +477221240, e-mail: kaniewscy@o2.pl
\end{abstract}

\begin{abstract}
Aim: The aim of the study was to assess the effectiveness of a patented preparation of sodium butyrate in the triglyceride matrix at reducing clinical symptoms and improving quality of life in patients with irritable bowel syndrome (IBS).

Material and methods: In this prospective multicenter clinical trial, we observed a total of 3000 non-hospitalized patients with confirmed IBS who were treated with sodium butyrate in a triglyceride matrix at a dosage of $150 \mathrm{mg}$ twice a day for 12 weeks. The primary outcome was to evaluate the efficacy of sodium butyrate at reducing the severity of clinical symptoms and improving quality of life in patients with IBS.

Results: A total of 2990 complete surveys were collected. A statistically significant improvement in severity of abdominal pain was noticed $(p<0.001)$. Moreover, flatulence, diarrhea, constipation, urgent pressure for bowel movements, nausea, and vomiting decreased significantly $(p<0.001)$. Most of the respondents $(93.90 \%)$ declared that they would continue the therapy and $88.9 \%$ would recommend using sodium butyrate to other IBS patients.

Conclusions: Sodium butyrate in the triglyceride matrix, as a postbiotic substance, may be effective in relieving the symptoms of IBS by modifying the intestinal microbiota.
\end{abstract}

\section{Introduction}

Irritable bowel syndrome (IBS) is the most common ( $11 \%$ of the total population) chronic disease, manifested by abdominal pain and bowel movement disturbances. The most important pathophysiological factors are disturbances in the regulation of the brain-intestine axis, increased visceral sensation, impaired motor and secretory function of the intestines, disturbances in the composition of the intestinal microbiota, and a history of infectious diarrhea (post-infectious irritable bowel syndrome). Psychiatric disorders occur in $70 \%-90 \%$ of patients [1-3].
According to the Rome IV criteria, diagnosis is based on the presence of abdominal pain coexisting with disturbances in the rhythm of bowel movements, the appearance of the stool or defecation. The following 4 forms are distinguished: with diarrhea, with constipation, mixed, and undefined [1-3]. Good cooperation with the patient is key in treatment. It is imperative to clarify the cause of the symptoms and to ensure that the disease is not serious. Dietary interventions are the first line of treatment, including a low-FODMAP diet [2].

Butyric acid (BA) is one of the short-chain fatty acids commonly found in the large intestine (including acetic acid and propionic acid). They show a strong trophic ef- 
fect on the intestinal epithelium. Sources of short-chain fatty acids are hexose oligomers with varying degrees of polymerization, indigestible carbohydrates (resistant starch, non-starch polysaccharides, inulin, oligofructose, lactose, and stachyose) and indigestible endogenous carbohydrates (mucins, chondroitin sulfate, and others). Butyric-acid-producing bacteria include Clostridioides sp., Eubacterium sp., and Fusobacterium sp. [4]. BA plays an important role in maintaining the correct $\mathrm{pH}$ level and protecting the intestinal mucosa against microbes. It is also used to stimulate the growth and maturation of intestinal epithelial cells, which results in the gradual reconstruction of diseased intestinal mucosa and the restoration of the physiological intestinal barrier [5]. BA stimulates the reabsorption of sodium and water and plays a role in maintaining the water-electrolyte balance. The pro-absorption properties of butyrate with respect to sodium absorption are in contrast to the antisecretory effect on chlorine release. BA inhibits the activity of the $\mathrm{Na}-\mathrm{K}-2 \mathrm{Cl}$ cotransporter (NKCC1). Accordingly, it can limit diarrhea by reducing water secretion into the intestinal lumen [6]. Due to its low stability and unpleasant odor, it was replaced with a sodium salt preparation.

There are several different products containing sodium butyrate on the Polish market. In 2017, all products containing sodium butyrate in oral forms were analyzed. The profile of butyrate release in the gastrointestinal (GI) tract was investigated and the highest concentration in the colon of $35-50 \%$ was found from microencapsulated preparations [7]. Data on the effectiveness of microencapsulated sodium butyrate, which can reduce symptoms and improve quality of life among IBS patients, are limited to studies in small groups of patients, so we decided to conduct this study.

\section{Material and methods \\ Study design}

The study was a multi-center cross-sectional study on the use of microencapsulated sodium butyrate in patients with IBS, conducted from January 1 to October 1 , 2019. A total of 3000 patients with confirmed IBS were enrolled in the study. Completed questionnaires were obtained from 2990 patients with IBS; the response rate was $99.7 \%$. During the study, appointments were made before the start of microencapsulated sodium butyrate therapy and after 12 weeks. The questionnaire included a few questions related to the diagnosis and severity of gastrointestinal symptoms.

The questionnaire for the first visit contained the following information:

1) the diagnosis - based on a positive response by a physician to the following: (1) IBS with dominant diarrhea; (2) dominant constipation; (3) with variable bowel movements; and (4) unclassified form.

2) the intensity of self-reported GI symptoms - based on a positive response to the following: (1) abdominal pain related to defecation, change in bowel movement frequency, change in stool consistency, postprandial and spontaneous; (2) discomfort in the abdominal cavity; (3) diarrhea; (4) constipation; (5) flatulence; (6) urgent pressure for bowel movements; (7) nausea; and (8) vomiting. The question used a scale from 0 (minimum score) to 10 (maximum score).

3) previous treatment - based on a positive choice of the following: (1) no treatment; (2) diet; (3) lifestyle modification; (4) antiemetics; (5) prokinetic drugs; (6) laxatives; (7) constipation drugs; (8) antibacterial drugs; (9) spasmolytic drugs; (10) pain medications; (11) anti-flatulence drugs; (12) psychotherapy; and (13) psychotropic drugs.

4) the frequency of abdominal pain - (1) never; (2) less frequently than 1 day a month; (3) 1 day a month; (4) 1 or 2 days a month; (5) 1 day a week; (6) more than 1 day a week; and (7) every day.

$5)$ the frequency of constipation (0-2 bowel movements per week): (1) never or rarely; (2) sometimes; (3) often; (4) most of the time; and (5) always.

6 ) the frequency of diarrhea ( $>3$ bowel movements per day): (1) never or rarely; (2) sometimes; (3) often; (4) most of the time; and (5) always.

The questionnaire at the second appointment included Questions 2, 3, 4, 5, and 6 from the questionnaire for the first visit and two additional ones: will the subjects continue microencapsulated sodium butyrate treatment (Yes/No) and would they recommend the treatment to other patients? (Yes/No/I don't know).

\section{Setting}

In this prospective multicenter study, each patient had a confirmed diagnosis of IBS based on the Rome IV criteria [8]. To be eligible, the participants had to meet all of the following criteria: (1) age $\geq 18$ years; (2) an IBS diagnosis based on the Rome IV criteria; (3) an independent decision of the doctor to change the current treatment to the supplemented patented product of sodium butyrate in the triglyceride matrix (Intesta), made in consultation with the patient; (4) a psychophysical state of health, which indicates compliance with medical recommendations. The exclusion criteria were (1) chronic mental illness; (2) alcohol and/or drug addiction; and (3) contraindications to the use of Intesta, which contains sodium butyrate.

\section{Statistical analysis}

Statistical analysis was conducted with Statistica software, version 13.0 (https://www.statsoft.pl/). Nom- 
Table I. Descriptive statistics of age $\left(^{+}\right)$and BMI $\left(^{\ddagger}\right)$ researchers by gender $\left({ }^{\dagger} p=0.192 ;{ }^{\ddagger} p<0.001\right)$

\begin{tabular}{|c|c|c|c|c|c|c|c|}
\hline \multirow{2}{*}{$\begin{array}{l}\text { Analyzed } \\
\text { variable }\end{array}$} & \multirow[t]{2}{*}{ M } & \multirow[t]{2}{*}{$\mathrm{Me}$} & \multicolumn{3}{|c|}{ Statistical parameter } & \multirow[t]{2}{*}{$95 \% \mathrm{Cl}$} & \multirow[t]{2}{*}{ Min.-max. } \\
\hline & & & $\mathrm{Q}_{1}-\mathrm{Q}_{3}(\mathrm{IQR})$ & SD & SE & & \\
\hline \multicolumn{8}{|c|}{ Age $\left(^{+}\right)$[years]: } \\
\hline Women & 49.03 & 48 & $38-60(22)$ & 15.01 & 0.34 & $48.36-49.71$ & $18-96$ \\
\hline Men & 49.78 & 49 & $40-60(20)$ & 14.23 & 0.44 & $48.91-50.65$ & $18-89$ \\
\hline Total & 49.22 & 49 & $38-60(22)$ & 14.78 & 0.27 & $48.69-49.76$ & $18-96$ \\
\hline \multicolumn{8}{|c|}{ BMI $\left(^{\ddagger}\right)\left[\mathrm{kg} / \mathrm{m}^{2}\right]:$} \\
\hline Women & 25.74 & 25.30 & $22.31-28.34(6.03)$ & 4.60 & 0.11 & $25.53-25.95$ & $14.22-48.89$ \\
\hline Men & 27.29 & 27.02 & $24.75-29.39$ (4.64) & 3.84 & 0.12 & $27.06-27.53$ & $15.61-62.98$ \\
\hline Total & 26.26 & 26.03 & $23.07-28.85$ (5.78) & 4.40 & 0.08 & $26.10-26.42$ & $14.22-62.98$ \\
\hline
\end{tabular}

inal variables are presented as $n$ (\% frequency of group), while continuous variables are presented as mean (SD) or median $(\mathrm{Q} 1 ; \mathrm{Q} 3)$, depending on the normality of data distribution. The normality of the data was verified with the Shapiro-Wilk test and was based on a visual assessment of histograms. The groups were compared with the $\chi^{2}$ test for dichotomous variables and with the $t$-test or Mann-Whitney $U$ test for continuous variables, as appropriate. All tests were two-sided, with $\alpha=0.05$.

\section{Results}

Completed questionnaires were obtained from 2990 patients with IBS; the response rate was $99.7 \%$. The study group was not similar with respect to sex distribution, as women comprised $65 \%$ and men 35\% of study participants. The mean age of the respondents was $49.22 \pm 14.78$ years. Most of the patients lived in cities with populations of up to 50000 . Patients with secondary education constituted over $40 \%$ of the sample. Over half (59.56\%) of the respondents were overweight or obese, as defined by a body mass index (BMI) of $>25.0 \mathrm{~kg} / \mathrm{m}^{2}$, which was statistically significantly more men $(73.45 \%)$ than women $(52.51 \%) ; p<0.001$ (Table I).

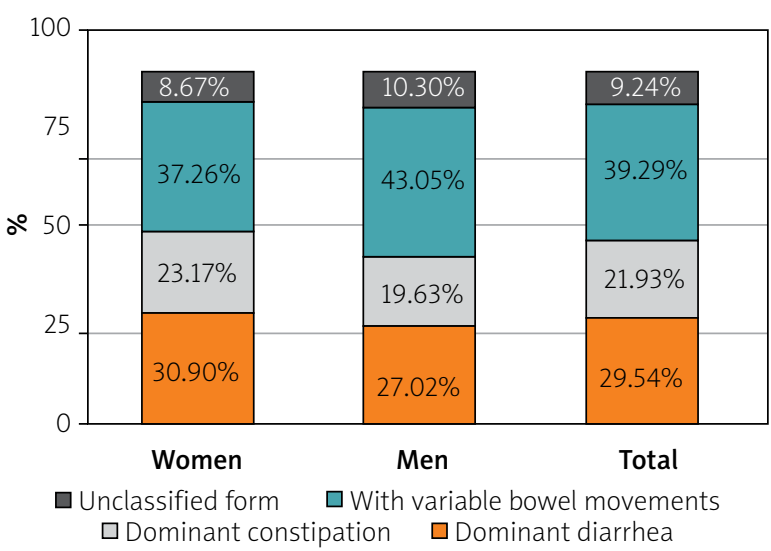

Figure 1. Forms of IBS in the cohort
Among the 2990 patients in the IBS cohort, the form with variable bowel movements was the most common (39.29\%), followed by the types with dominant diarrhea (29.54\%), dominant constipation (21.93\%), and unclassified (9.24\%). The most common form of IBS in both sexes was IBS with variable bowel movements (37.26\% of women vs. $43.05 \%$ of men) (Figure 1 ).

The severity of Gl symptoms was measured on a scale of 0 to 10 , where 0 was a lack of Gl symptoms and 10 was the most severe of $G$ l symptoms. The mean scores given by IBS patients for their symptoms were as follows: flatulence -6.18 points; abdominal pain 5.11 points; diarrhea -4.94 points; constipation -4.41 ; urgent pressure for bowel movements -4.14 ; nausea 1.64 points; and vomiting -0.87 points (Figure 2 ). The most frequently used drugs in the treatment of IBS-related symptoms before sodium butyrate therapy were antispasmodics (in $43.87 \%$ of cases) (Figure 3 ).

The patients' assessment of the severity of their IBS symptoms in the context of quality of life was given on a scale from 0 to 10 points, where 0 points means their symptoms do not affect the quality of life and 10 points means the symptoms make it impossible to perform a given activity. The activities assessed in the questionnaire and the average scores were as follows: quality of life 6.61 ; social life -6.43 ; professional work -6.08 ; household duties - 5.8; and hobbies -5.62 points (Figure 4).

After 12 weeks of microencapsulated sodium butyrate therapy, a statistically significant improvement in the severity of GI symptoms was noted at the follow-up appointments. The severity of abdominal pain (related to defecation, a change in the frequency of bowel movements, a change in the consistency of stools, postprandial pain, and spontaneous pain) improved significantly $(p<0.001)$. Moreover, flatulence, diarrhea, constipation, urgent pressure for bowel movements, nausea, and vomiting also decreased significantly ( $p<0.001)$ (Figure 5).

The 12 -week intake of sodium butyrate was assessed by the patients in four categories - treatment 


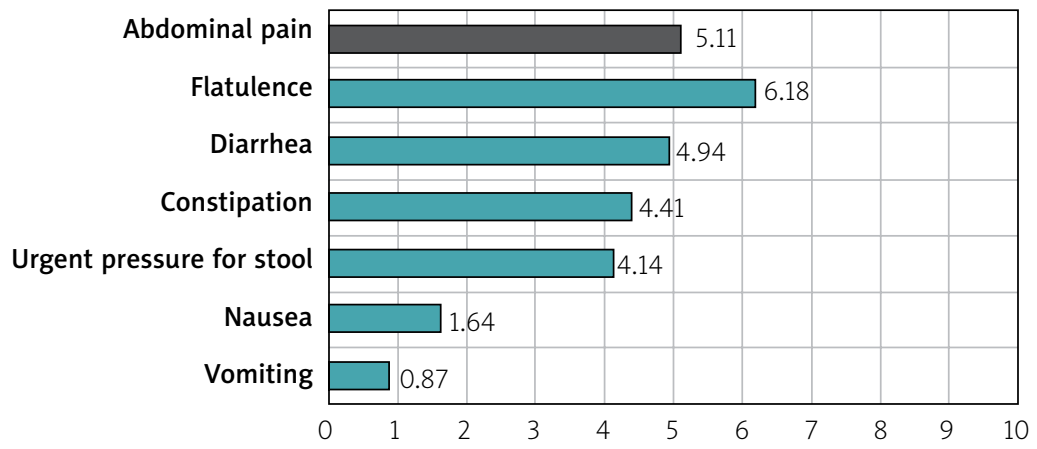

Figure 2. Severity of selected gastrointestinal symptoms in IBS patients

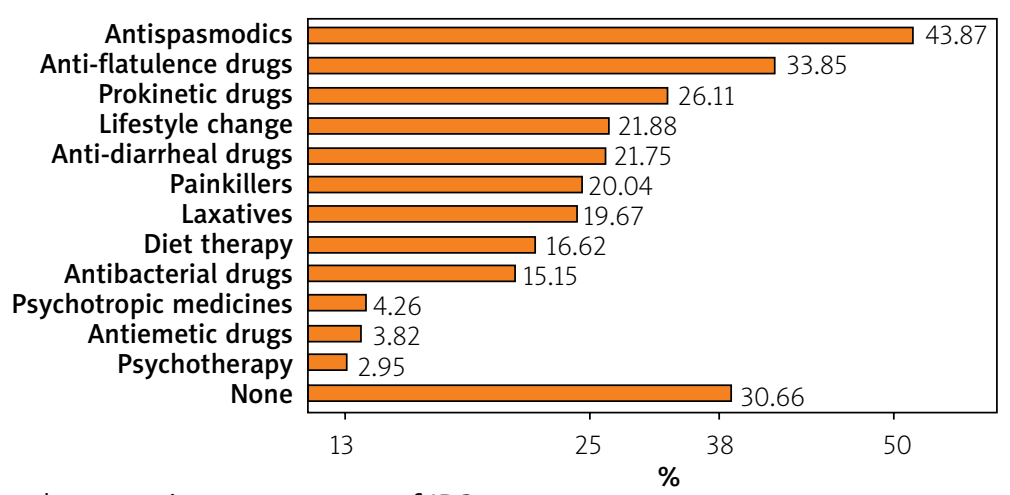

Figure 3. Previous therapeutic management of IBS

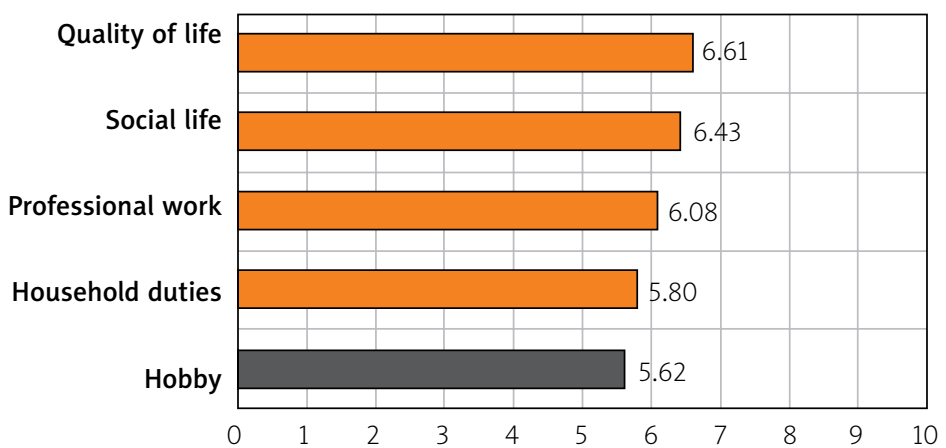

Figure 4. Assessment of the nuisance of IBS symptoms in terms of the patients' quality of life

tolerance, costs of therapy, convenience of use, and efficacy - and was compared with the treatment they were receiving prior to the study. Treatment tolerance was higher for butyrate than for the previous treatment $(p<0.001)$. Sodium butyrate at a dosage of $150 \mathrm{mg}$ twice daily was rated better than previous treatments (7.85 vs. 5.3; $p<0.001)$. Treatment costs were estimated to be lower (7.78 vs. $4.81 ; p<0.001)$. Treatment effectiveness was also significantly higher (7.4 vs. 3.39; $p<0.001$ ) (Figure 6). The quality of life, social life, professional work, household duties, and hobbies or interests was compared between the beginning of the study and the end of the study. Quality of life improved from 6.61 to $3.66(p<0.001)$. Social life also improved significantly, from 6.43 to $3.73(p<0.001)$. Professional work also improved, from 6.08 to 3.41 ( $p<0.001)$. Comfort increased as well within the household duties, from 5.8 to 3.31 ( $p<0.001)$. The ability to pursue hobbies also improved, from 5.62 to $3.84(p<0.001)$ (Figure 7).

A strong majority $(93.90 \%)$ of the respondents declared their willingness to continue the therapy. Treatment was discontinued by 182 patients $(6.1 \%$ of the entire cohort), 121 of whom cited the lack of a therapeutic effect. Most of the respondents (88.90\%) would recommend using microencapsulated sodium butyrate to other IBS patients.

\section{Discussion}

To our knowledge, our IBS patient cohort is the largest so far among Polish studies. In 2012, Ziółkowski 


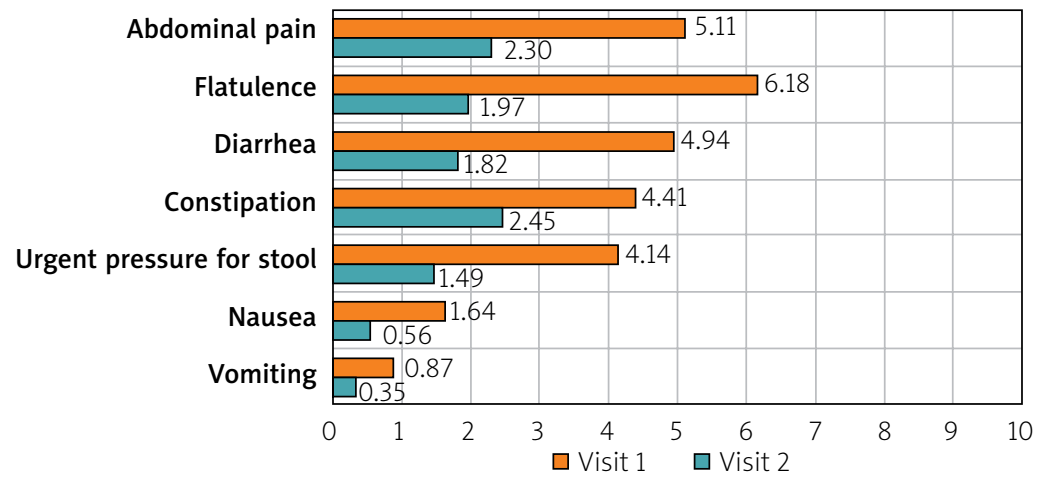

Figure 5. Comparison of the severity of GI symptoms of IBS among patients before and after BA intervention

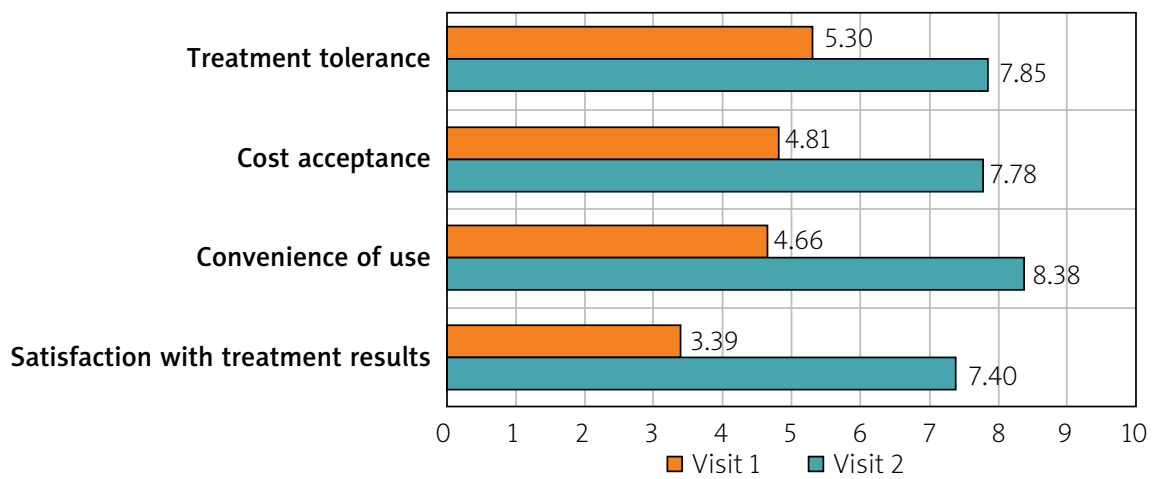

Figure 6. Comparison of sodium butyrate treatment vs. previous treatment

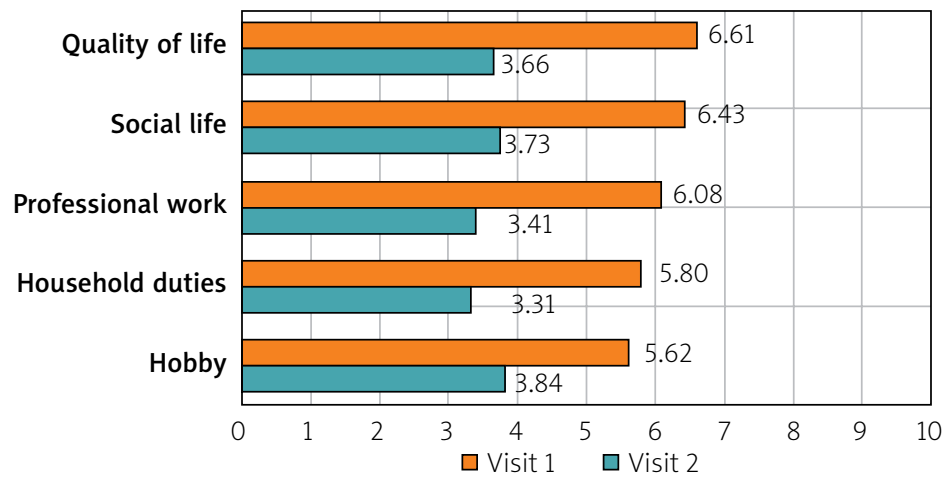

Figure 7. Patients' quality of life compared between baseline and at the end of the study

et al. published data on the prevalence of common Gl disorders, such as dyspepsia, gastroesophageal reflux disease, IBS, constipation, and bloating, in the Polish population. A total of 850 patients were interviewed, and $13 \%$ of them had had IBS symptoms. All of the above-mentioned complaints were slightly more common in diabetic patients, but the difference was not significant. Diabetes is associated with overweight and obesity, in particular; this was evident in our study, as $59.56 \%$ of the respondents were overweight or obese [9]. In the Polish Guidelines for the conduct of IBS, Pietrzak et al. pointed out that IBS is $14 \%$ for women and
$8.9 \%$ for men. In our study, there was a similar finding: women suffered more from IBS $(65 \%$ of women vs. $35 \%$ of men) [10].

Tarnowski et al. evaluated the effect of orally administered protected sodium butyrate on the clinical improvement and quality of life in patients with IBS. A sample of 59 people were examined and divided into 2 groups. The first group received standard treatment with trimebutine or mebeverine, while the second group received the same standard treatment with protected sodium butyrate for 6 weeks. Both groups experienced a significant improvement in clinical symptoms and 
quality of life. Discomfort and abdominal pain and defecation rhythm disturbances were significantly reduced after the therapy with additional protected sodium butyrate in comparison with the standard therapy. The patients' quality of life was also significantly better in the group receiving the protected sodium butyrate. The authors concluded that IBS might be associated with a lower production of BA by microorganisms, mainly related to diet modification and the elimination (more or less consciously) of butyrogenic products and disturbances to the intestinal microbiota. Consequently, this leads to impaired regulatory mechanisms of which BA is an essential element. There was no evidence that BA deficiency may be directly related to the pathogenesis of IBS, but it is known that a lack of BA might exacerbate the disease symptoms. In summary, a 6-week therapy of protected sodium butyrate improved quality of life and alleviated clinical symptoms in patients with IBS. According to the authors of the study, it was worth including sodium butyrate in the diet of patients with IBS [11]. In our study, we used a different questionnaire, a more complex one which assessed the severity and frequency of clinical symptoms. The research group was also larger, which makes our findings more reliable. Sodium butyrate was effective in reducing abdominal discomfort and pain and contributed to the normalization of the defecation rhythm. However, from the patients' point of view, the most important aspect for them is their quality of life, which also improved as a result of the therapy.

Banasiewicz et al. included 66 patients who had been treated with standard pharmacological therapies for at least 3 months. The cohort was divided into 2 groups. The first group included patients using sodium butyrate as an adjunct to therapy, and the second group received a placebo. Clinical assessment - based on a validated questionnaire measuring the severity of selected IBS symptoms, a closed questionnaire measuring the frequency of symptoms, and a closed-question questionnaire measuring the subjective assessment of the improvement in the patients' symptoms - was carried out 4 and 12 weeks after the start of therapy. The results of the study showed that after 4 weeks there was a significant reduction in pain during defecation in the sodium butyrate group. After 12 weeks of treatment, the percentage of patients reporting spontaneous abdominal pain, postprandial pain, defecation disturbances, an urge to defecate, a change in stool consistency, and constipation decreased. Mucous in the stool was a parameter that remained unchanged. Sodium butyrate as an adjunct to standard pharmacological therapy may reduce the incidence of clinical symptoms of IBS [12]. The study was randomized and placebo-controlled, which favorably influenced the results. Unfortunately, the answers to the questions were not statistically significant, probably because the cohort was too small. The results of our study also showed a beneficial effect on these ailments.

The standard therapy of sodium butyrate consists in taking the substance daily at a dosage of 150-300 $\mathrm{mg} /$ day. Data on the use of higher dosages of sodium butyrate are currently available, and studies have been conducted in an animal model [13]. A study in mice showed improvement in epithelial function. Sodium butyrate was administered in drinking water (about $11 \mathrm{~g} /$ day) [14]. In the conclusions, the authors presented the theory that the use of such high doses of sodium butyrate may be associated with an action intended to prevent chronic diseases [15].

In other studies based on an animal model, a beneficial effect of sodium butyrate on colon cancer cells was found [16]. This phenomenon is referred to in the literature as the butyrate paradox. It also seems that due to the above-mentioned properties it is justified to use sodium butyrate in anti-cancer prophylaxis. Moreover, it may be useful in the treatment of obesity, metabolic syndrome, cardiovascular diseases, hereditary diseases, chronic immune-related disorders, and neurodegeneration [17].

Despite the fact that the study was conducted on a relatively large number of patients, it has the limitation of not being randomized.

\section{Conclusions}

According to our results, sodium butyrate in the triglyceride matrix seems to be effective at reducing $\mathrm{Gl}$ symptoms and improving quality of life among IBS patients, though placebo-controlled randomized trials are required.

\section{Conflict of interest}

Partially sponsored by a scientific grant from Bioton.

\section{References}

1. Mulak A, Smereka A, Paradowski L. Novelties and modifications in the Rome IV criteria. Gastroenterol Klin 2016; 2: 1-10.

2. Pietrzak A, Skrzydło-Radomańska B, Mulak A, et al. Guidelines on the management of irritable bowel syndrome. Gastroenterology Rev 2018; 13: 259-88.

3. Aziz I, Tornblom H, Palsson O, et al. How the change in IBS Criteria from Rome III to Rome IV impacts on clinical characteristics and key pathophysiological factors. Am J Gastroenterol 2018; 113: 1017-25.

4. Kien C, Blauwiekel R, Bunn J, et al. Cecal infusion of butyrate increases intestinal cell proliferation in piglets. J Nutr 2007; 137: 916-22. 
5. Chapman M, Grahn M, Boyle M, et al. Butyrate oxidation is impaired in the colonic mucosa of sufferers of quiescent ulcerative colitis. Gut 1994; 35: 73-6.

6. Feng W, Wu Y, Chen G, et al. Sodium butyrate attenuates diarrhea in weaned piglets and promotes tight junction protein expression in colon in a GPR109A-dependent manner. Cell Physiol Biochem 2018; 47: 1617-29.

7. Banasiewicz T. Badanie porównujące profil uwalniania się w jelicie aktualnie dostępnych na rynku polskim produktów zawierających maślan sodu. Med Fakt 2017; 4 (37).

8. Drossman D. Functional gastrointestinal disorders: history, pathophysiology, clinical features, and Rome IV. Gastroenterology 2016; 150: 1262-79.

9. Ziółkowski BA, Pacholec A, Kudlicka M, et al. Prevalence of abdominal symptoms in the Polish population. Gastroenterology Rev 2012; 7: 20-5.

10. Pietrzak A, Skrzydło-Radomańska B, Mulak A, et al. Guidelines on the management of irritable bowel syndrome. Gastroenterology Rev 2018; 13: 259-88.

11. Tarnowski W, Borycka-Kiciak K, Kiciak A, et al. Outcome of treatment with butyric acid In irritable bowel syndrome - preliminary report. Gastroenterol Prakt 2013; 1: 43-8.

12. Banasiewicz T, Krokowicz $t$, Stojcev Z, et al. Microencapsulated sodium butyrate reduces the frequency of abdominal pain in patients with irritable bowel syndrome. Colorectal Dis 2013; 15: 204-9.

13. Banasiewicz T, Domagalska D, Borycka-Kiciak K, et al. Determination of butyric acid dosage based on clinical and experimental studies - a literature review. Gastroenterology Rev 2020; 15: 119-25.

14. Goverse G, Molenaar R, Macia L, et al. Diet-derived short chain fatty acids stimulate intestinal epithelial cells to induce mucosal tolerogenic dendritic cells. J Immunol 2017; 198: 2172-81.

15. McIntyre A, Gibson P, Young G. Butyrate production from dietary fiber and protection against large bowel cancer in rat model. Gut 1993; 34: 386-91.

16. Canani RB, Di Costanzo M, Leone L. The epigenetic effects of butyrate: potential therapeutic implications for clinical practice. Clin Epigen 2012; 4: 4.

17. Andoh A, Bamba T, Sasaki P. Physiological and anti-inflammatory roles of dietary fiber and butyrate in intestinal functions. J Parent Ent Nutr 1999; 23: 70-3.

Received: 4.12.2021

Accepted: 15.12 .2021 